\title{
Over-the-scope clip placement is effective rescue therapy for severe acute upper gastrointestinal bleeding
}

Authors

Institution
Matthew Skinner, Juan P. Gutierrez, Helmut Neumann, C. Mel Wilcox, Chad Burski, Klaus Mönkemüller

Basil I. Hirschowitz Endoscopic Center of Excellence, Department of Gastroenterology, University of Alabama, Birmingham, Alabama, United States received 18. December 2013 accepted after recision 4. January 2014

\section{Bibliography}

Dol http://dx.doi.org/

10.1055/s-0034-1365282

Published online: 7.3.2014

Endoscopy International Open

2014; 02: E37-E40

(c) Georg Thieme Verlag KG

Stuttgart · New York

E-ISSN 2196-9736

\section{Corresponding author}

Klaus Mönkemüller, MD, PhD,

\section{FASGE}

Department of

Gastroenterology

University of Alabama

1617 Hamilton Court

Birmingham, AL 35243

United States

klaus1@uab.edu

moenkemueller@yahoo.com
Background and study aim: The novel over-thescope clip (OTSC) allows for excellent apposition of tissue, potentially permitting hemostasis to be achieved in various types of gastrointestinal lesions. This study aimed to evaluate the usefulness and safety of OTSCs for endoscopic hemostasis in patients with upper gastrointestinal bleeding in whom traditional endoscopic methods had failed. Patients and methods: A retrospective case series of all patients who underwent placement of an OTSC for severe recurrent upper gastrointestinal bleeding over a 14-month period was studied. Outcome data for the procedure included achievement of primary hemostasis, episodes of recurrent bleeding, and complications.

Results: Twelve consecutive patients (67\% men; mean age 59, range 29-86) with ongoing upper gastrointestinal bleeding despite previous endoscopic management were included. They had a

\section{Introduction \\ $\nabla$}

Current endoscopic methods to provide hemostasis for acute upper gastrointestinal bleeding include clips, injection, argon plasma coagulation (APC), Hemospray, and monopolar electrocoagulation $[1,2]$. When repeated endoscopic therapy fails to achieve hemostasis, treatment options have previously been limited to angiography with embolization or surgery [3-5]. The novel over-the-scope clip (OTSC; Ovesco, Tübingen, Germany), which is made of a superelastic nickel titanium alloy, provides excellent tissue apposition, potentially permitting hemostasis to be achieved in various types of gastrointestinal lesions [611]. The aim of this study was to evaluate the clinical usefulness and safety of the use of OTSCs for endoscopic hemostasis in patients with acute nonvariceal upper gastrointestinal bleeding in whom traditional endoscopic methods had failed. mean ASA score of 3 (range 2-4), a mean hemoglobin of $7.2 \mathrm{~g} / \mathrm{dL}$ (range 5.2-9.1), and shock was present in $75 \%$ of patients. They had all received packed red blood cells (mean 5.1 units, range 212). The etiology of bleeding was: duodenal ulcer $(n=6)$, gastric ulcer $(n=2)$ Dieulafoy lesion $(n=2)$, anastomotic ulceration $(\mathrm{n}=1)$, Mallory-Weiss tear $(n=1)$. Hemostasis was achieved in all patients. Rebleeding occurred in two patients 1 day and 7 days after OTSC placement. There were no complications associated with OTSC application. Conclusions: OTSC use represents an effective, easily performed, and safe endoscopic therapy for various causes of severe acute gastrointestinal bleeding when conventional endoscopic techniques have failed. This therapy should be added to the armamentarium of therapeutic endoscopists.

\section{Patients and methods}

$\nabla$

This was an observational, open-label, retrospective, single-arm case series conducted at a large tertiary care hospital from October 2012 to November 2013. All of the procedures were performed by one endoscopist (K.M.). Inclusion criteria were recurrent gastrointestinal bleeding after the failure of two sessions of endoscopic therapy. Exclusion criteria included pregnancy, patients aged less than 18 years, or failure to provide informed consent. Patient demographic, clinical, and laboratory data, including American Society of Anesthesiologist's preoperative risk (ASA) score, hemoglobin level, hematocrit, number of units of packed red cells transfused prior to the procedure, presence of clinically evident shock number of attempted prior endoscopic procedures, lesion type, location, size, Forrest classification of the lesion, technical and clinical success, complications, and follow-up clinical outcome were reviewed. 

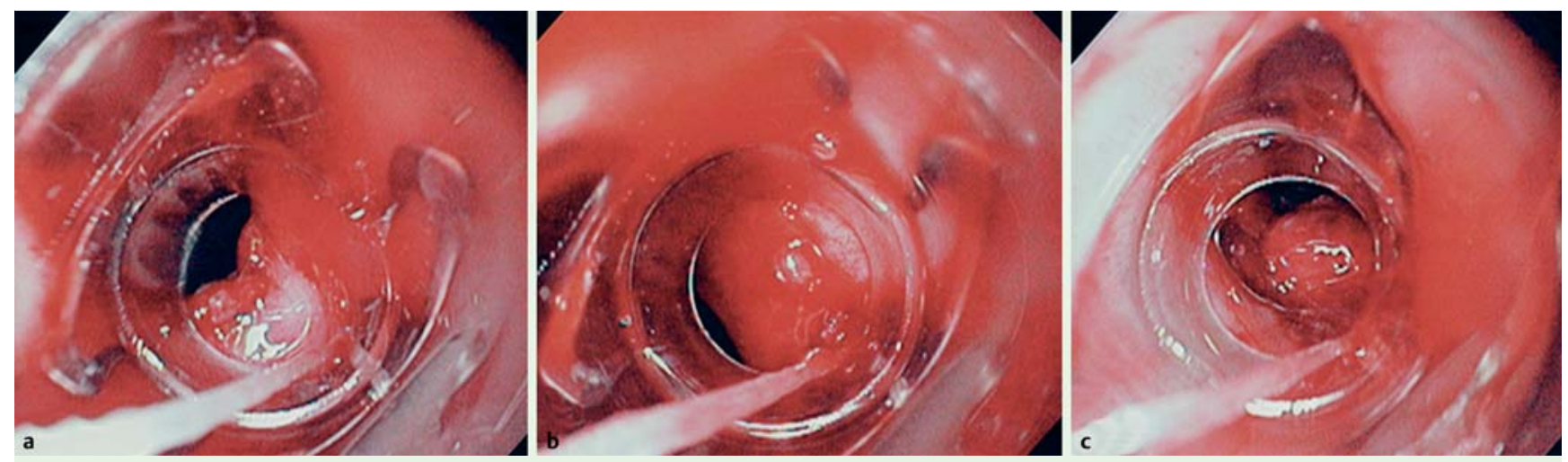

Fig. 1 A bleeding duodenal ulcer that was treated by the application of an over-the-scope clip (OTSC). a The lesion in the posterior wall was closely inspected. b Before the OTSC device was deployed, the cap of the device was carefully placed perpendicular (on top of or en face) to the ulcer and the bleeding vessel. c The OTSC has been successfully deployed.

Technical success was defined as deployment of the OTSC onto the target lesion, with technical failure defined as misplacement of the OTSC. Clinical success was defined as the achievement of hemostasis, with clinical failure defined as recurrent bleeding within 30 days, which included hematemesis, melena, or a drop in hemoglobin/hematocrit with unstable cardiovascular parameters (systolic blood pressure $<90 \mathrm{mmHg}$ or heart rate $>100$ beats per minute). If rebleeding was suspected, emergency esophagogastroduodenoscopy (EGD) was performed. Possible complications included those related to sedation, endoscopy, or deployment of the OTSC.

The OTSC system ("bear claw") is a CE and FDA-approved device for the closure of mucosal defects, endoscopic marking, and hemostasis. In this study, the OTSC was used according to the manufacturer's instructions. The OTSC system was loaded onto a Q160 or Q180 Olympus gastroscope (Olympus America, Melville, New York, USA) and then introduced into the upper gastrointestinal tract under standard direct visualization, similar to when advancing a loaded six-shooter device for treatment of esophageal varices. The bleeding lesion was located and the gastroscope was maneuvered toward it ( Fig. 1a). Once the bleeding lesion had been located in the center of the cap ( $\bullet$ Fig. 1b), the scope and cap were pushed toward the tissue, full suction was applied to the tissue, so that the lesion was fully engulfed inside the transparent cap before the clip was released ( $\bullet$ Fig. 1c, $\bullet$ Fig. 2). No additional devices, such as the tissue grasping forceps (Twin Grasper; Ovesco, Tübingen, Germany), were used. In two patients who had post-bulbar ulcers, a wire, or a wire placed inside a feeding tube, was advanced into the distal duodenum ( $\bullet$ Fig. 3). The scope was then advanced alongside the wire. This measure helped to prevent small-bowel luminal occlusion, which has previously been reported as a major adverse event [8].

Data are presented using descriptive statistics.

\section{Results}

$\nabla$

During the 14-month period, 12 patients ( 8 men, 4 women; mean age 59, range 29-86) were evaluated. They had a mean ASA score of 3 (range 2-4) and a mean hemoglobin of $7.2 \mathrm{~g} / \mathrm{dL}$ (range 5.2-9.1). All patients had bled significantly: $75 \%$ of the patients initially presented with hemodynamic shock and all of the patients had received packed red blood cells, with the mean number of units transfused being 5.1 (range 2-12). All patients had failed hemostatic therapy using traditional endoscopic methods. The mean number of endoscopies that had been performed prior to OTSC was 2.1 (range $2-3$ ). Patients had bleeding for the following reasons: duodenal ulcer $(n=6)$, gastric ulcer $(n=$ $2)$, Dieulafoy lesion $(n=2)$, anastomotic ulceration $(n=1)$, and Mallory-Weiss tear $(n=1)$.

Hemostasis was achieved in all patients. Rebleeding occurred in two patients 1 day and 7 days after OTSC placement. Subsequently, the patient with a Mallory - Weiss tear was successfully treated with an injection of saline/epinephrine and the placement of conventional clips. The patient with gastrojejunal anastomotic ulcers was readmitted with melena 7 days after placement of the OTSC. Repeat EGD showed active bleeding from a large, circumferential ischemic anastomotic ulcer. No endoscopic intervention was undertaken and the patient proceeded to radiological embolization and then to surgery for reconstruction of the anastomosis. There were no complications associated with the application of OTSCs. The demographic, clinical, and endoscopic findings for the 12 patients are summarized in $\bullet$ Table 1 .

\section{Discussion}

\section{$\nabla$}

This study shows that the OTSC system readily provides hemostasis and can function as a "rescue therapy" in patients with severe nonvariceal upper gastrointestinal bleeding in whom prior endoscopic therapies have failed. Historically these patients would have required more invasive procedures such as embolization or surgery, $[4,5]$ with embolization being relatively contraindicated in patients with renal disease because of the need to administer intravenous contrast.

Our study adds to the growing literature on the utility of the OTSC system in the treatment of upper gastrointestinal bleeding. The largest multicenter study, which included three tertiary centers in the United States, reported on a total of seven patients who had been treated with OTSCs for gastrointestinal bleeding, which probably reflected the initial experience and sporadic use of the OTSC for gastrointestinal bleeding [8]. Therefore, additional data such as ours are important in the evaluation of the potential utility of OTSCs for gastrointestinal bleeding.

In contrast to other studies, we have also provided a description of the technical steps necessary for the use of this device in acute nonvariceal upper gastrointestinal bleeding. We believe that this short, but clear, description of the OTSC system enables the 

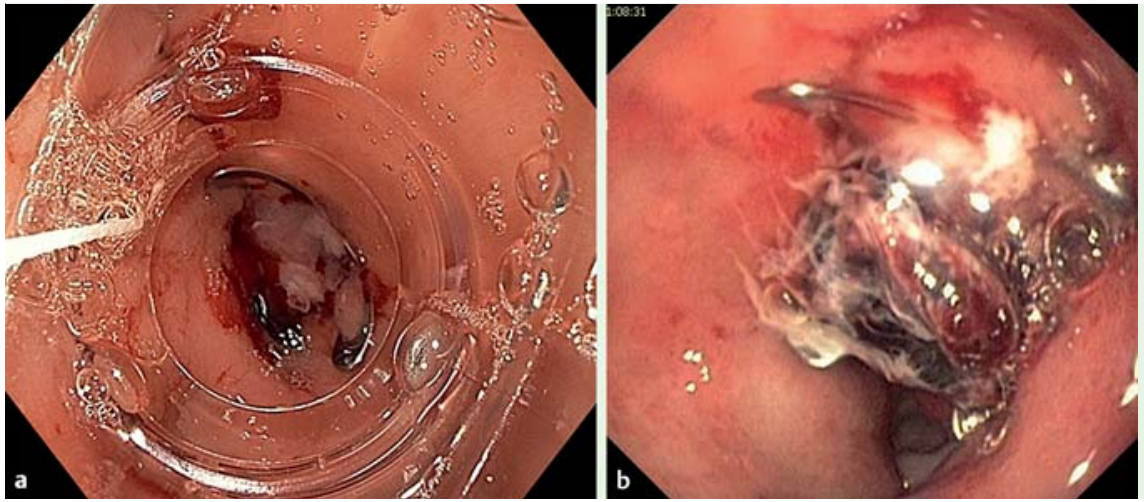

Fig. 2 Typical images of deployed over-the-scope clips (OTSCs) showing: a the amount of tissue entrapped within the clip; $\mathbf{b}$ clots, fibrin, and tissue entrapped within the clip.
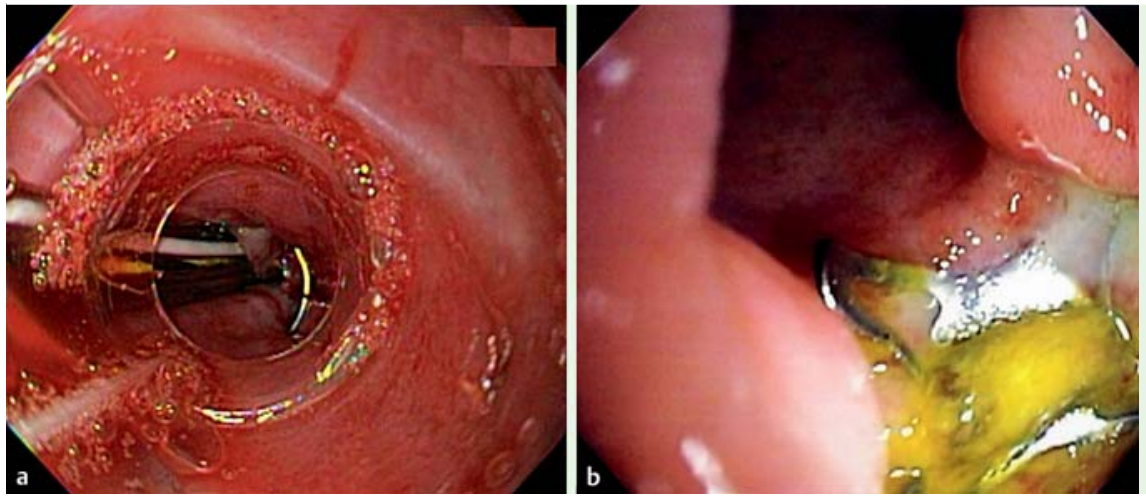

Fig. 3 a In patients where the lumen was poorly visualized, a feeding tube with a wire within it was advanced into the distal duodenum to maintain luminal patency and aid visualization. $\mathbf{b}$ The clips typically stay in position for several weeks, but in this patient adequate fibrosis and ulceration is shown within the entrapped tissue 72 hours after deployment.

endoscopist to better comprehend the utility and applicability of this endoscopic hemostatic device. We want to emphasize that we used endoscopes that are available as standard. In the largest North American series published to date, all seven patients were treated with therapeutic gastroscopes [8]. Although the use of large-diameter scopes with larger suctioning and working channels could have advantages when managing larger lesions, most endoscopy units have standard gastroscopes, so our data may be more applicable to general practice.

Although first-line clipping devices have achieved excellent rates of hemostasis, the rebleeding rates are clinically significant [3, $10,12]$. The OTSC system utilizes a very contractile, superelastic nickel titanium alloy, which provides tissue apposition that is far superior to that of traditional clipping. Based on our experience and the published data, the OTSC system appears promising for the treatment of bleeding lesions with large-diameter visible vessels or those located in awkward positions, such as the greater curvature of the stomach or the posterior duodenal wall, which may not always be amenable to treatment with standard endoscopes and endoscopic devices $[1,5,13]$. The transparent cap can be directed onto lesions that are in awkward positions, and once the cap is in place, suction can be applied to draw larger volumes of tissue into the cap. This tissue is then squeezed by the clip, further compressing the centrally located vessels. On the basis of our experience, we are convinced that the tissue-apposition capabilities make the OTSC an essential part of the endoscopist's armamentarium for the control of bleeding lesions that are considered to be high risk or difficult to treat using other endoscopic methods.

We want to address potential limitations of our study. First, it is retrospective and therefore has the limitations of any such study. Second, it reflects the experience of a tertiary-care center; however, the scopes used are present in most hospitals.
In summary, the OTSC system represents an effective, easily performed, and safe endoscopic therapeutic modality for various causes of severe acute gastrointestinal bleeding. We were able to demonstrate its usefulness as a "rescue therapy," that provided hemostasis for both large lesions and lesions that were in awkward positions. This endoscopic hemostatic therapy should be considered when conventional endoscopic techniques have failed. We recommend interventional endoscopists add this therapy to their armamentarium. Early results demonstrate efficacy of the OTSC system in the treatment of nonvariceal gastrointestinal bleeding; however, more prospective clinical trials are needed to demonstrate its superiority over traditional clips and closure devices. 


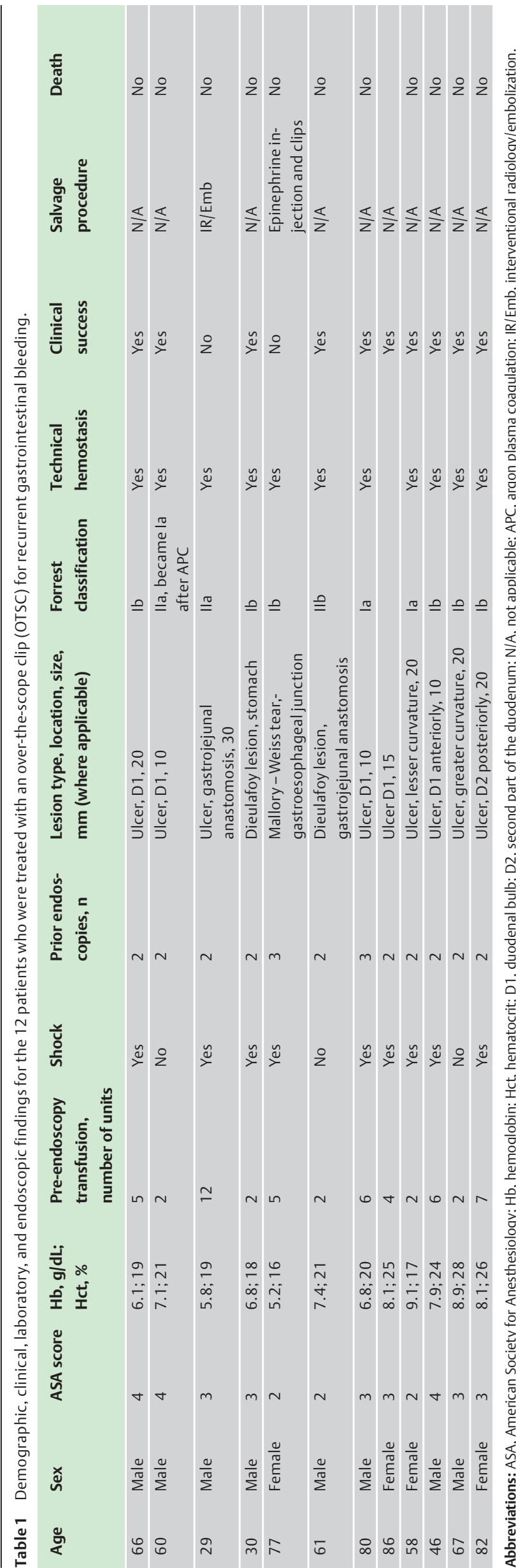

Competing interests: $\mathrm{KM}$ has received a one-time travel grant from Ovesco, USA in the amount of $\$ 1500$ to attend a meeting at UEGW Berlin 2013 which he gave a lecture on techniques for endoscopic closure and hemostasis using various endoscopic devices. None of the other authors have any conflicts of interest to declare.

\section{References}

1 SungJY, Chung SC, Lo KK et al. Heater-probe treatment of bleeding peptic ulcers. Surg Endosc 1988; 2: 234-236

2 Aabakken L. Current endoscopic and pharmacological therapy of peptic ulcer bleeding. Best Pract Res Clin Gastroenterol 2008; 22: $243-$ 259

3 Anastassiades CP, Baron TH, Wong Kee Song LM. Endoscopic clipping for the management of gastrointestinal bleeding. Nat Clin Pract Gastroenterol Hepatol 2008; 5: 559-568

4 Rösch J, Dotter CT, Brown MJ. Selective arterial embolization. A new method for control of acute gastrointestinal bleeding. Radiology 1972; 102: $303-306$

5 Millat B, Hay JM, Valleur P et al. French Associations for Surgical Research. Emergency surgical treatment for bleeding duodenal ulcer: oversewing plus vagotomy versus gastric resection, a controlled randomized trial. World J Surg 1993; 17: 568 - 573 (discussion 574)

6 von Renteln D, Rudolph HU, Schmidt A et al. Endoscopic closure of duodenal perforations by using an over-the-scope clip: a randomized, controlled porcine study. Gastrointest Endosc 2010; 71: 131 - 138

7 Pohl J, Borgulya M, Lorenz D et al. Endoscopic closure of postoperative esophageal leaks with a novel over-the-scope clip system. Endoscopy 2010; 42: 757-759

8 Baron TH, Song LM, Ross A et al. Use of an over-the-scope clipping device: multicenter retrospective results of the first U.S. experience (with videos). Gastrointest Endosc 2012; 76: 202 -208

9 Sandmann M, Heike M, Faehndrich M. Application of the OTSC system for the closure of fistulas, anastomosal leakages and perforations within the gastrointestinal tract. Z Gastroenterol 2011; 49: 981 -985

10 Banerjee S, Barth BA, Bhat YM et al. ASGE Technology Committee. Endoscopic closure devices. Gastrointest Endosc 2012; 76: 244-251

11 Mönkemüller K, Toshniwal J, Zabielski M. Utility of the "bear claw", or over-the-scope clip (OTSC) system, to provide endoscopic hemostasis for bleeding posterior duodenal ulcers. Endoscopy 2012; 44: (Suppl 2 UCTN) E412 - E413

12 Sung JJ, Tsoi KK, Lai LH et al. Endoscopic clipping versus injection and thermo-coagulation in the treatment of non-variceal upper gastrointestinal bleeding: a meta-analysis. Gut 2007; 56: $1364-1373$

13 Mönkemüller K, Neumann $H$, Bellutti $M$ et al. Use of a colonoscope to perform endoscopic therapy in patients with active bleeding from posterior duodenal and gastric ulcers. Endoscopy 2009; 41: E93 -E94 